\title{
Disfunção Sistólica Aguda de Ventrículo Esquerdo após Drenagem de Derrame Pericárdico
}

\author{
Fábio Branco de Araújo Brauner, Cristine Erdmann N unes, Rogério Fabra, Alberto Riesgo, \\ Luiz Gustavo Thomé \\ Santa Maria, RS
}

Paciente com timoma e função sistólica normal do ventrículo esquerdo desenvolveu tamponamento cardíaco, revertido por pericardiocentese. Após quatro dias, foi submetida à exérese do tumor e, em aproximadamente uma semana da drenagem, apresentou disfunção sistólica aguda e grave do ventrículo esquerdo, que melhorou com terapia venosa, normalizando o quadro em três dias.

\section{Transient Left Ventricular Systolic Dysfunc- tion after Pericardial Effusion Drainage}

A patient with a thymoma and initially normal ventricular systolic function developed cardiac tamponade, which was relieved by pericardiocentesis. After four days, the tumor was removed and, one week after the relief of tamponade, she developed severe left ventricular systolic dysfunction, that recovered in three days with venous therapy.

Arq Bras Cardiol, volume 69 (nº 6), 421-423, 1997

O tamponamento cardíaco pode ocasionar diminuição da função sistólica do ventrículo esquerdo (VE) por redução da perfusão coronária, com melhora após sua drenagem ${ }^{1-3}$. Entre as causas de tamponamento cardíaco encontram-se os tumores torácicos e, entre eles, o timoma ${ }^{4}$.

É raro o aparecimento de disfunção sistólica aguda e grave após a pericardiocentese, sendo relatados na literatura apenas dois casos de tumores de mama com derrame pericárdico, que evoluíram com grave acometimento da função sistólica após drenagem do tamponamento, e que obtiveram melhora, espontaneamente, no decorrer de duas semanas ${ }^{5}$.

Descrevemos um caso onde a descompensação sistólica ocorreu após nove dias da pericardiocentese, com evolução à normalização da função em três dias.

\section{Relato do Caso}

Mulher, branca, 61 anos, foi hospitalizada com queixas de dispnéia, fraqueza e considerável emagrecimento. Ao exame físico tinha pressão arterial de $100 / 60 \mathrm{mmHg}$, freqüência cardíaca de $130 \mathrm{bpm}$, freqüência respiratória de 28 movi-

Hospital de Caridade Dr. Astrogildo de Azevedo - Santa Maria

Correspondência: Cristine Erdmann Nunes - Rua Faria Santos, 125 - 90670-150

- Porto Alegre, RS

Recebido para publicação em 2/6/97

Aceito em 8/10/97 mentos por minuto e pulso paradoxal de $15 \mathrm{mmHg}$. Foram observados: turgência jugular, hepatomegalia (fígado com $18 \mathrm{~cm}$ de comprimento) sem ascite e edema de membros inferiores $++++/ 4$. A ausculta cardíaca revelava bulhas hipofonéticas, sem outras alterações significativas.

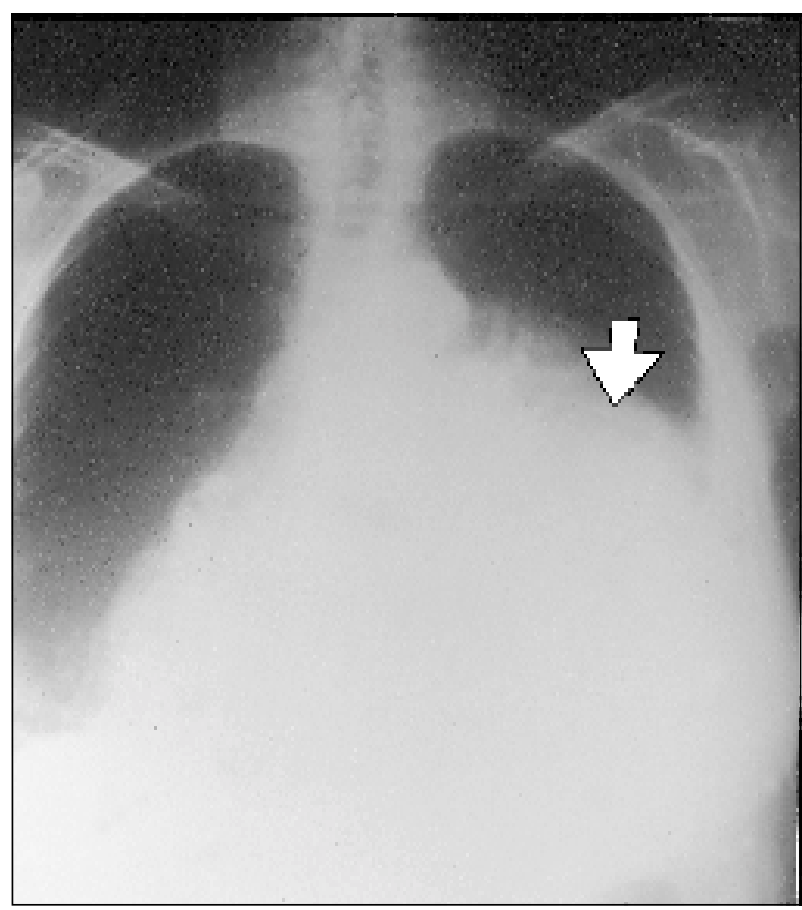

Fig. 1 - Radiografia de tórax AP: massa no hemitórax esquerdo. 
Trazia um radiograma de tórax antigo que mostrava massa no mediastino anterior, confirmada no novo exame radiológico feito na internação (fig. 1).

A paciente realizou tomografia computadorizada de tórax (fig. 2) que evidenciou lesão expansiva em mediastino anterior à esquerda, em contato com a aorta, hilo pulmonare pericárdio, além de um conteúdo hipodenso em saco pericárdico (derrame pericárdico). Massa que, posteriormente, foi diagnosticada como um timoma. O ecocardiograma (ECO) confirmou o derrame pericárdico e mostrou a função sistólica normal. A pericardiocentese foi realizada com drenagem de $600 \mathrm{ml}$ de líquido amarelo citrino, havendo subseqüente melhora do tamponamento cardíaco. Em quatro dias, a paciente foi encaminhada à cirurgia para a exérese do timoma, evoluindo bem no pós-operatório.

Feito ECO de controle três dias após a cirurgia, que mostrou mínimo derrame pericárdico residual e função contrátil do VE preservada (FE Teicholtz: 76\%; espessamento do pericárdio visceral com mínimo derrame; cavidades com dimensões normais - átrio esquerdo (AE) $38 \mathrm{~mm}$, volume diastólico final do VE 43mm, diâmetro sistólico final do VE24mm, ventrículo direito(VD) $12 \mathrm{~mm}$ ).

Em dois dias, a paciente iniciou quadro clínico de insuficiência cardíaca esquerda, apresentando dispnéia paroxística noturna, ortopnéia e $3^{\mathrm{a}}$ bulha. Repetido o ECO (fig. 3), foi evidenciada disfunção contrátil grave do $\mathrm{VE}$ (fração de ejeção $(\mathrm{FE})<30 \%$ ). A paciente apresentou parada respiratória durante o exame, sendo entubada e colocada em ventilação mecânica.

Uma nova tomografia de tórax, feita com o objetivo de auxiliar na elucidação diagnóstica, mas não conclusiva, mostrou apenas regressão do derrame pericárdico e pequena opacidade na região do timoma ressecado.

Com a finalidade de excluir um possível infarto agudo

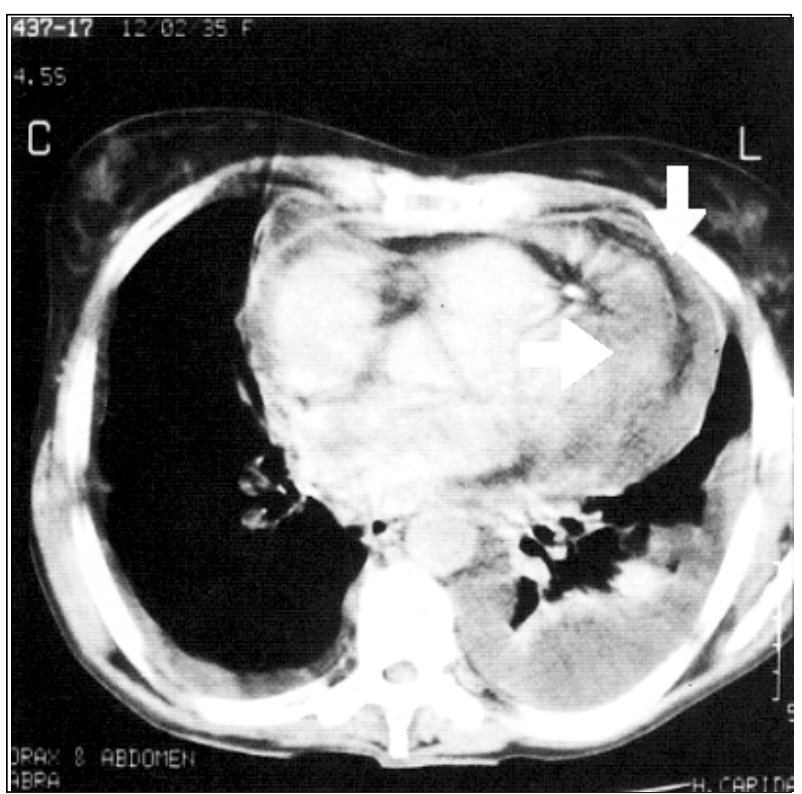

Fig. 2 - Tomografia computadorizada de tórax: lesão expansiva no mediastino anterior esquerdo e derrame pericárdico

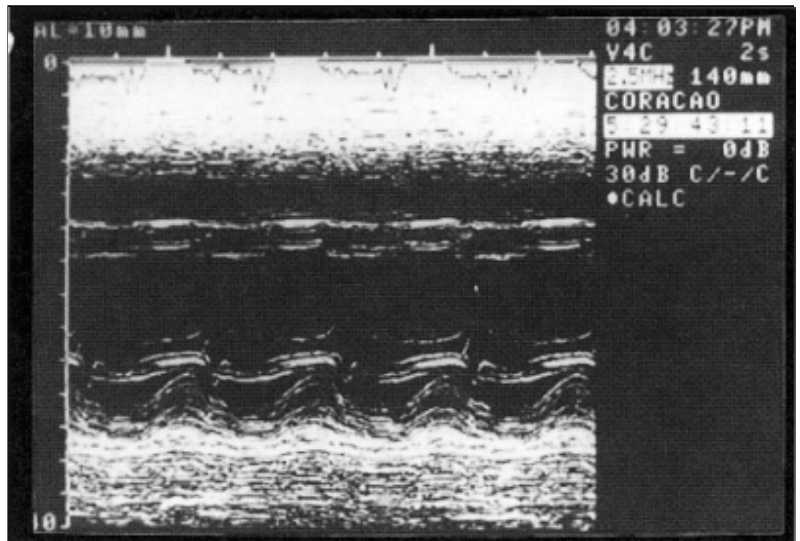

Fig. 3 - Ecocardiograma: disfunção contrátil grave do ventrículo esquerdo (VE) (fração de ejeção <30\%); acinesia ântero-septo-apical e ínfero-lateral; contratilidade preservada apenas nas porções basais do VE; dimensões das cavidades: diâmetro diastólico do VE46,4mm, diâmetro sistólico do VE39,4mm, fração de encurtamento $15 \%$, ventrículo direito (VD) $13 \mathrm{~mm}$, átrio esquerdo (AE) $38 \mathrm{~mm}$, espessura diastólica do septo $8 \mathrm{~mm}$, espessura diastólica da parede posterior do VE $8 \mathrm{~mm}$, índice de Teicholtz 32\%, volume diastólico VE 99,3ml, volume sistólico VE 67,5ml, volume de ejeção $31,8 \mathrm{ml}$.

do miocárdio, como causa dessa disfunção, realizou-se cateterismo cardíaco, que apontou coronárias normais, além de VE com hipocinesia difusa grave. As enzimas cardíacas eram normais.

A paciente foi internada na UTI, sendo administrados dobutamina e diurético de alça em pequenas doses, já que se apresentava em hipotensão grave.

Após três dias do início do quadro de insuficiência cardíaca esquerda foi repetido ECO, com melhora significativa da função sistólica (FE 59\%). As medidas das cavidades foram: AE 35mm, VD 20mm, diâmetro diastólico final VE $45 \mathrm{~mm}$, diâmetro sistólico final VE $31 \mathrm{~mm}$, espessura diastólica do septo $10 \mathrm{~mm}$, espessura diastólica da parede posterior do VE 10mm, fração de encurtamento sistólico 31\%, volume diastólico final do VE $92,45 \mathrm{ml}$, volume sistólico final do VE37,92ml, volume de ejeção 54,5ml.

\section{Discussão}

Estudos têm mostrado que o tamponamento cardíaco pode piorar a função sistólica do VE e que a função sistólica, geralmente, melhora após drenagem do tamponamento ${ }^{1-3}$. Descrevemos o caso de uma paciente com timoma e derrame pericárdico maligno, cuja disfunção sistólica grave se desenvolveu após a pericardiocentese, evoluindo favoravelmente, após instituição de terapêutica com medicação endovenosa e cuidados em unidade de tratamento intensivo. A paciente apresentava função sistólica prévia normal.

Na literatura, os dados sobre este assunto são limitados. Foram encontrados dois casos de pacientes com câncer de mama que desenvolveram o quadro clínico em questão ${ }^{5}$. Na busca de explicações para essa disfunção sistólica, Wolfe e Edelman ${ }^{5}$ descreveram várias teorias, entre elas, que tal disfunção estaria oculta pela redução no tamanho das câmaras e pela taquicardia associada durante o tampo- 
namento, ou a disfunção estivesse sido engatilhada por alterações hemodinâmicas que se seguiram à remoção do líquido pericárdico e alívio do tamponamento. Também foi descrito que o sistema nervoso autônomo estaria previamente ativado durante o tamponamento e que a remoção desse estímulo simpático, através da pericardiocentese, poderia levar à disfunção do $\mathrm{VE}^{3}$.
Disfunção aguda de VE após drenagem pericárdica

O conhecimento da possibilidade de ocorrer uma disfunção sistólica grave, após drenagem de derrame pericárdico, é importante, pois, além de ser um fato não usual na prática médica, traz implicações clínicas, já que a realização de quimioterapia - com agentes potencialmente cardiotóxicos - fica prejudicada, em paciente com disfunção sistólica grave ${ }^{5}$.

\section{Referências}

1. Frank MJ, Nadimi M, Lesniak LJ, Hilmi KI, Levinson GE- Effects of cardiac tamponade on myocardial performance, blood flow and metabolism. Am J Physiol 1971; 220: 179-85.

2. Wechsler AS, Auerbach BJ, Graham TC, Sabinston DC - Distribution of intramyocardial blood flow during pericardial tamponade. J Thorac Cardiovasc Surg 1974; 68: 847-56.
3. Martins JB, ManuelWJ, Marcus ML, KerberRE-Comparative effects of catecholamines in cardiac tamponade: experimental and clinical studies. Am J Cardiol 1980; 46: 59-66.

4. Shishido M, Yano K, Ichiki H, Yano M - Pericarditis as the initial manifestation of malignant thymoma. Chest 1994; 106: 313-14.

5. Wolfe MW,Edelman ER-Transient systolic dysfunction after relief of cardiac tamponade. Ann Int Med 1993; 119: 42-4. 